\title{
The Thermographic Phosphor Labkit
}

\author{
Mark M. McKeever*, Michael R. Cates*, Stephen W. Allison*, David L. \\ Beshears*, Alfred Akerman*, Matthew B. Scudiere*, and James E. Parks† \\ *EMCO, 1813 Chestnut Grove, Knoxville, TN. 37932 \\ $\dagger$ Department of Physics, University of Tennessee, 1408 Circle Drive, Knoxville, TN. 37996
}

\begin{abstract}
The thermographic phosphor (TGP) Labkit is a flexible instrument for students beyond the first year and is also a useful laboratory tool for physics and research and development. It aids in teaching and demonstrating a number of useful concepts and skills. Students will learn important aspects of optical physics and spectroscopy. By producing fluorescence and measuring its characteristics versus temperature, students will become familiar with atomic energy levels, lifetimes, radiative and non-radiative transitions, spectral bands and linewidth, spectral distribution, and absorption. In addition, measurements involve optics, electro-optics, and electronic instrumentation. The Labkit contains an ultraviolet light emitting diode of $365 \mathrm{~nm}$ that illuminates a thin phosphor layer. The phosphor adheres to a copper plate in intimate contact with a Peltier heater/cooler that covers 0 to $100{ }^{\circ} \mathrm{C}$. A thermocouple attaches to the copper plate to monitor temperature. The Labkit allows for controlling the pulse duration of the LED and the rate of repetition. Representative signals from 10 to $55^{\circ} \mathrm{C}$ are presented and the resulting well behaved lifetime versus temperature plot. Results of separate measurements at $10{ }^{\circ} \mathrm{C}$ indicate repeatability. Detector linearity is demonstrated over a factor of eight in detector gain. Practice and familiarity with common laboratory and industry tools such as thermocouples, Peltier devices, and photomultiplier detectors also contribute to the educational objectives. Finally, in the course of exercising the instrument, the student will learn the practical skill of temperature measurement and control.
\end{abstract}

Keywords: Energy Levels, Lifetimes, Fluorescence, Temperature, Non-contact Temperature Measurements.

\section{INTRODUCTION}

Temperature is one of the most important attributes of physical systems, and its measurement is critical to many aspects of scientific research and development. Some situations requiring the thermographic phosphor approach include vibrating or moving surfaces, difficult to access surfaces, hazardous locations, high electro-magnetic fields, and the like. Typical examples include centrifuges, turbine engine components, high speed motors, radioactive or very high temperature environments, or vibrating or moving production machinery. In many situations, it is also necessary to measure temperature remotely, without direct contact, because of difficulties of access, intervening heated air or other gases, or movement of the component to be measured. Such instances are covered in a number of survey articles. ${ }^{1-6}$

Phosphors are fine white or pastel colored powders commonly used for fluorescent lighting, LED lighting, $\mathrm{X}$-ray imaging, and displays. When illuminated by a proper source a phosphor efficiently converts the incident energy to its characteristic fluorescence or phosphorescence. The Labkit utilizes a phosphor, lanthanum oxysulfide activated with europium, $\mathrm{La}_{2} \mathrm{O}_{2} \mathrm{~S}: \mathrm{Eu}$, commonly used for X-ray applications whose emission at room temperature originates from three $\mathrm{Eu}^{3+}$ excited electronic energy levels. These are designated ${ }^{5} \mathrm{D}_{2},{ }^{5} \mathrm{D}_{1}$ and ${ }^{5} \mathrm{D}_{0}$ and the transitions terminate in any of several ${ }^{7} \mathrm{~F}$ states resulting in many emission bands from the blue to deep red. This phosphor exhibits a striking temperature dependence in this temperature range and is easily stimulated by an inexpensive ultraviolet LED. A segment of the emission is shown in Fig. 1 for several temperatures, T, for ${ }^{5} \mathrm{D}_{2}$ bands. The ${ }^{5} \mathrm{D}_{1}$ and ${ }^{5} \mathrm{D}_{0}$ bands do not exhibit significant temperature dependence below about 100 ${ }^{\circ} \mathrm{C}$ and $200{ }^{\circ} \mathrm{C}$, respectively.

TGPs have emissions that can be measured in various ways to determine temperature. The most common method has been to measure the fluorescence intensity as a function of time following pulsed illumination and extract the characteristic lifetime, $\tau$, of the emission.

$$
I(t)=I_{0} e^{-\frac{t}{\tau}}+b .
$$

$\mathrm{I}_{0}$ is the initial intensity of the luminescence at $\mathrm{t}=0$. In practice there will be a background signal originating from any of a variety of sources including detector dark current and/or room light leakage for example. Typically, a TGP will be relatively insensitive to temperature below a certain temperature at which it "turns on," that is, begins to have other deexcitation routes competitive in probability with the 
photon emission. At increasing temperatures the lifetime decreases rapidly until it becomes too short to be measured with electronics involved. That range of superb temperature sensitivity is typically several hundred degrees centigrade.

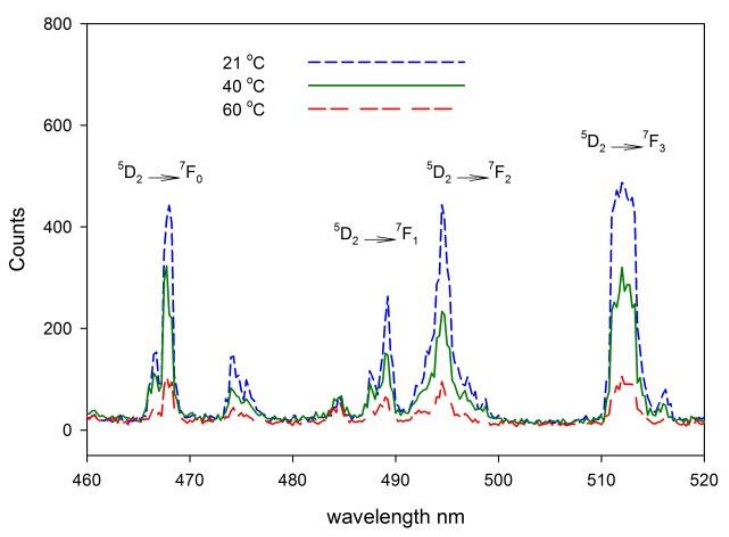

FIGURE 1. $\mathrm{La}_{2} \mathrm{O}_{2} \mathrm{~S}:$ Eu spectra between 460 and $520 \mathrm{~nm}$ at 21,40 , and $60{ }^{\circ} \mathrm{C}$.

A more detailed description that includes energy levels and the theory of temperature dependence is given by Parks $^{7}$.

\section{DESCRIPTION}

For calibrations near room temperature and to clearly illustrate the phosphor thermometry (PT) method, the Labkit contains the measurement components and basic software to convert fluorescent signals to temperature. The resulting signal is usually recorded by a laboratory oscilloscope, the digital signal transferred to a computer for signal analysis. Figure 2 is a photograph of the Labkit with an oscilloscope.

The Labkit contains an LED interrogation source, a sample holder for a standard $\mathrm{La}_{2} \mathrm{O}_{2} \mathrm{~S}$ :Eu phosphor that can be varied in temperature from about $-5^{\circ} \mathrm{C}$ to about $100^{\circ} \mathrm{C}$, a thermocouple to measure the sample temperature, narrow band filters for selecting desired wavelengths, and a photomultiplier detector to record the fluorescence signal. This is seen in FIGURE 3.

The Labkit provides control of the sample temperature, pulse width and repetition rate of the interrogating light source, an internal electronic trigger output for various uses such as triggering an oscilloscope, and an external trigger input so that external devices can pulse the light source or trigger the oscilloscope.

Note that the oscilloscope in Fig. 2 shows two traces. The higher (yellow) one is a typical fluorescence signal as a function of time, with an input impedance of $50 \mathrm{ohms}$. The lower (blue) signal is the voltage versus time applied to a pulser which drives the LED source. During this pulse the LED emits light and when the pulse switches off the LED light switches off. Typically a series of these pulses is sent to the LED producing a repeated illumination striking the phosphor sample up to hundreds of times per second.

Note that the fluorescence signal begins with the onset of the pulser signal, increases to a higher value, then begins to decrease exponentially after the LED switches off. If the LED pulse width were sufficiently wide the fluorescence signal would reach a maximum value and remain there until the LED switched off. It is apparent from the two signals that the time profile of both the fluorescence increase and decrease can be used to determine the characteristic lifetime of emission. That lifetime can be used to infer temperature. The Labkit is designed primarily for use in a laboratory environment, so that various TGPs and

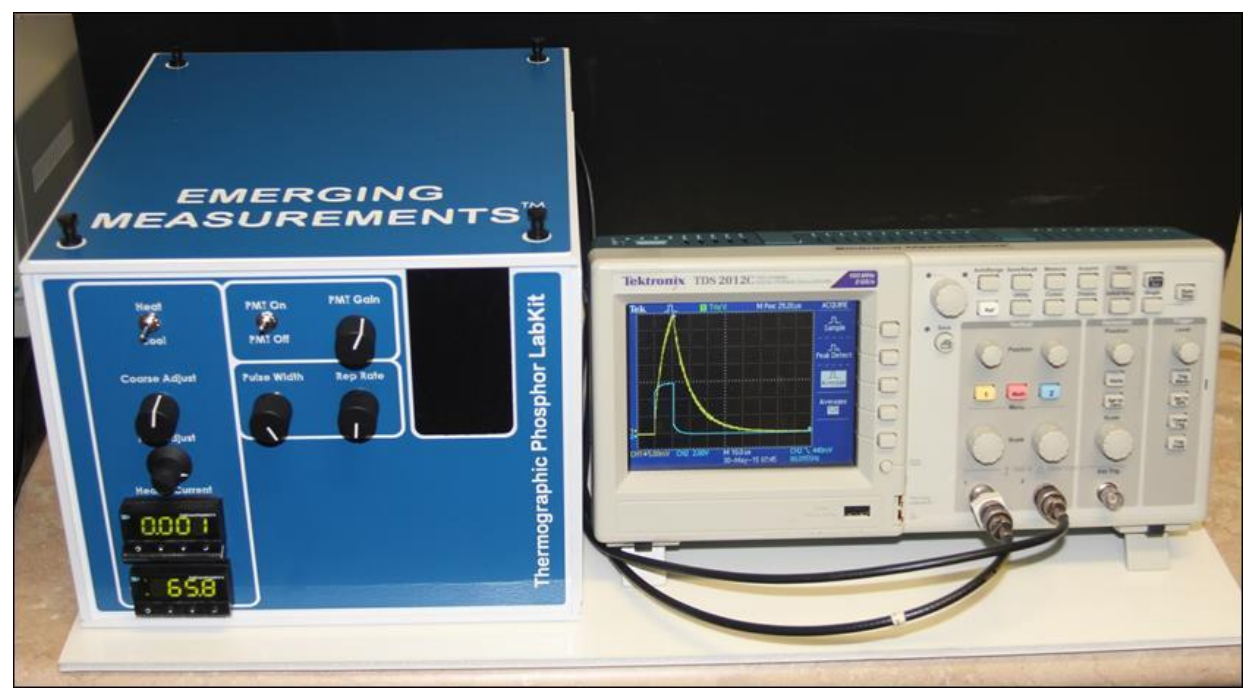

FIGURE 2. Labkit external package with digital oscilloscope. 


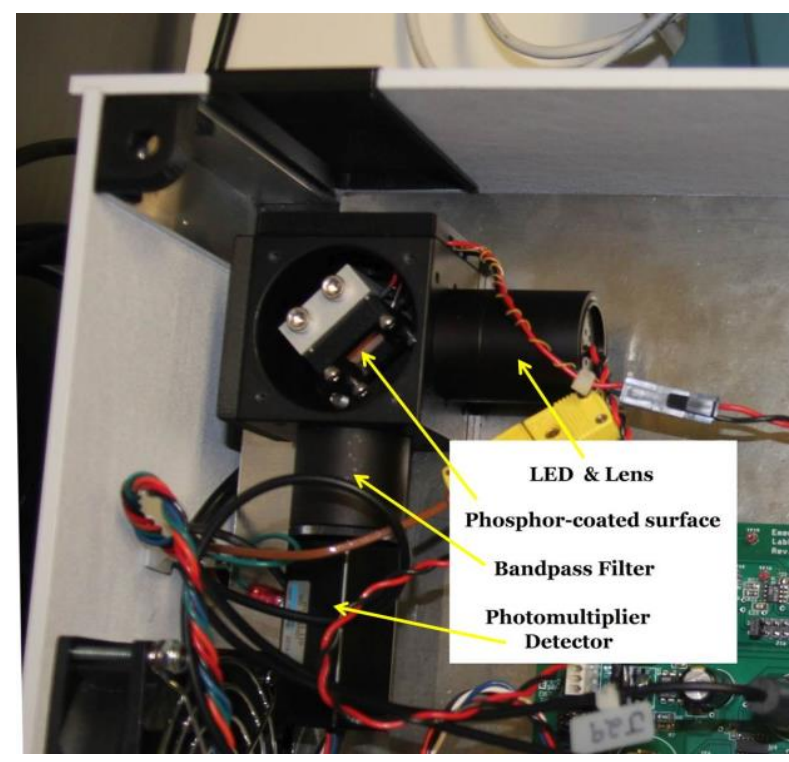

FIGURE 3. Optical arrangement inside Labkit.

LED sources can be evaluated for temperature response. The operation manual guides the user through the necessary aspects of its use. In addition, however, the Labkit can, in certain situations, be used for a temperature measurement application outside the laboratory. Different modifications of the system can be produced by EMCO for the special needs of the user. Typical among these modifications are provisions for an external interrogation source such as a laser; provisions for an external detector system, such as one involving fiber optics; or specialized sample holders for larger or more easily exchanged phosphor samples. Software for determining lifetime is provided. However, the teacher and/or students may choose their own approach to the analysis.

\section{RESULTS}

FIGURE 4 depicts signals from 10 to $55^{\circ} \mathrm{C}$ in five degree increments using the Labkit. The oscilloscope was set to provide a 128 -waveform running average. The LED turns on at about $-40 \mu \mathrm{s}$ in the figure where it is observed that the emission quickly begins to rise. At the coolest temperature, $10{ }^{\circ} \mathrm{C}$, the emission is still building up when at a time of about zero on this scale, the LED terminates and the emission promptly decreases exponentially. The buildup can be modelled as:

$$
I(t)=A_{\infty}\left(1-e^{-\frac{t}{\tau}}\right)
$$

Where $A_{\infty}$ is the limit of amplitude after many lifetimes, which, it may be seen also decreases with temperature and could be another feature that could be explored.

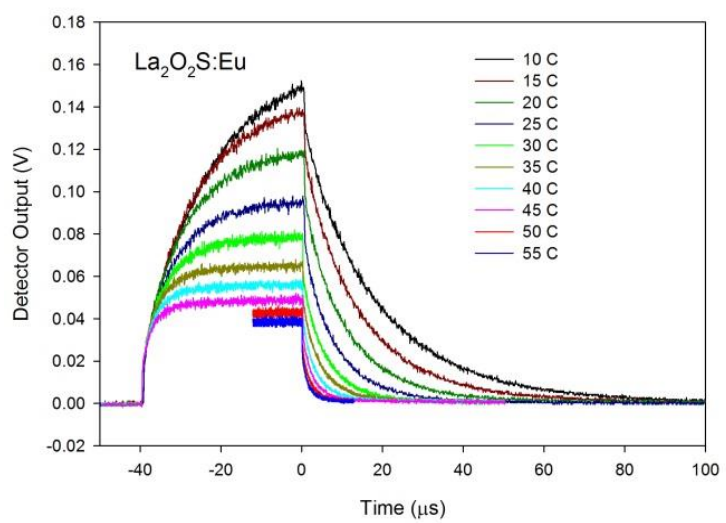

FIGURE 4. Fluorescence versus time showing temperature buildup while LED is on and decay of lifetime when LED terminated. From top to bottom, temperature ranges from 10 to $55^{\circ} \mathrm{C}$.

FIGURE 5 shows the logarithm of the curves of Fig FIGURE 4. It is clear that to a first approximation that the decay is linear in semi log space. Therefore from a fit to this data, an unambiguous lifetime can be determined. The estimated decay time for the $10{ }^{\circ} \mathrm{C}$ and $55^{\circ} \mathrm{C}$ curves are depicted which are $20 \mu$ s and 1.8 $\mu \mathrm{s}$, respectively.

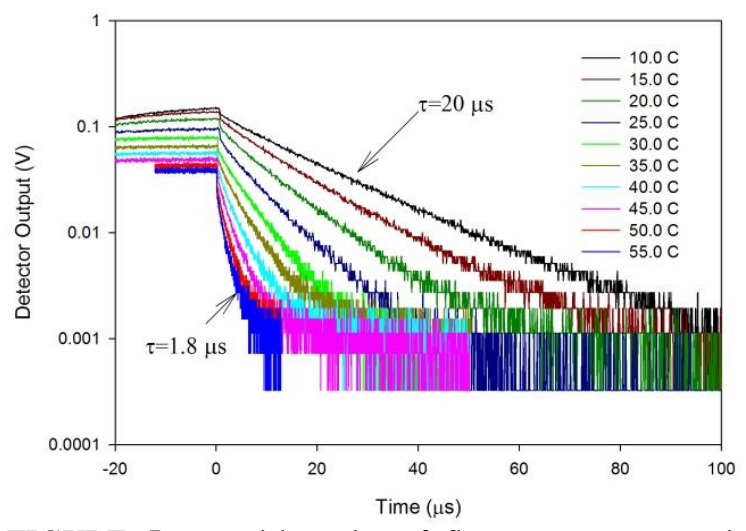

FIGURE 5. Semi-log plot of fluorescence versus time. From top to bottom, temperatures ranges from 10 to $55^{\circ} \mathrm{C}$.

A plot of temperature versus lifetime is shown in FIGURE 6. Estimated error bars are shown in red for 15,30 , and $50^{\circ} \mathrm{C}$.

FIGURE 7 depicts two signals at $10{ }^{\circ} \mathrm{C}$. The first was acquired at the beginning of a series of measurements up to $55{ }^{\circ} \mathrm{C}$ and back down to $10{ }^{\circ} \mathrm{C}$. The second signal was the last measurement of the series and was taken almost $2 \frac{1}{2}$ hours later, the end of the test. The two signals overlap quite well. A semilog plot of a segment, the inset in the figure, likewise illustrates the significant degree of repeatability. 


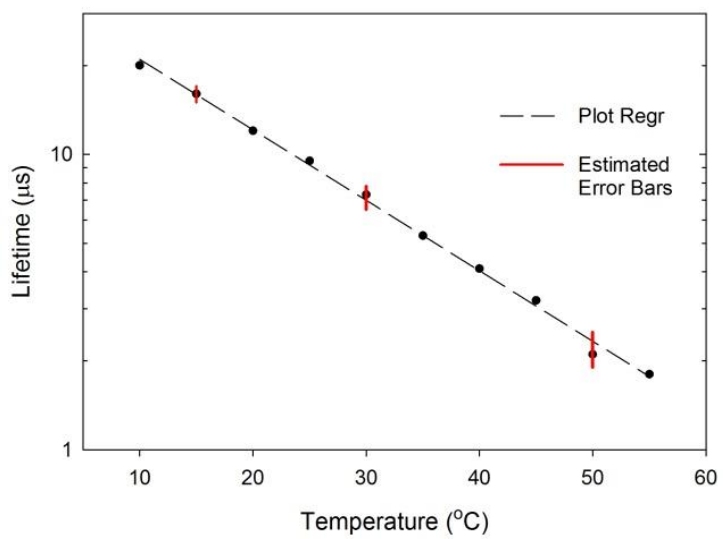

FIGURE 6. Temperature versus lifetime.

FIGURE 8 shows signals for several different gain settings of the photomultiplier tube detector such that the relative signal sizes are 1,2, 4 and 8 . The inset depicts a segment of the logarithm of each of these signals. The average lifetime of the four measurements was $9.71 \mu \mathrm{s}$ which corresponds to $22.5+/-0.2{ }^{\circ} \mathrm{C}$ according to the calibration data. The thermocouple indicated $21.7{ }^{\circ} \mathrm{C}$. The duty cycle was $20 \%$. Photomultipliers can respond nonlinearly to large signals. This measurement establishes that in this case it is not a significant effect.

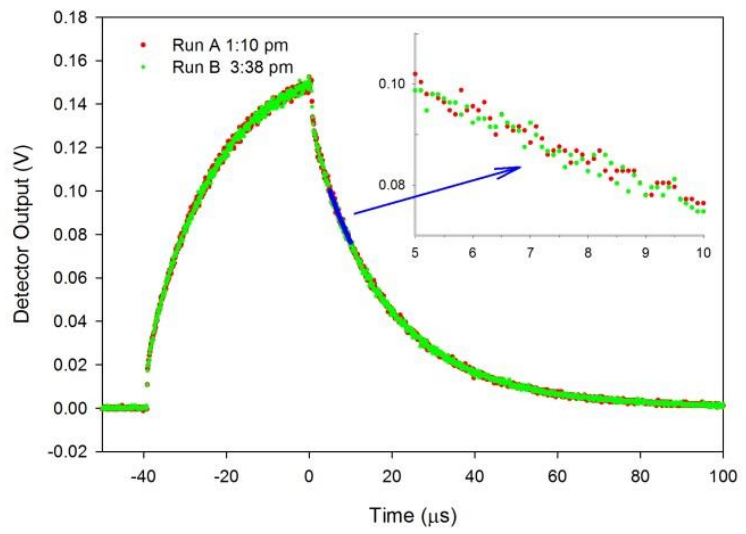

FIGURE 7. Data taken at $10{ }^{\circ} \mathrm{C}$ at different times after a temperature cycle.

\section{CONCLUSIONS}

The Labkit provides the means to generate and to detect luminescence from the electronic excited energy levels of the activator $\mathrm{Eu}^{3+}$ in $\mathrm{La}_{2} \mathrm{O}_{2} \mathrm{~S}$. Demonstrated here is the temperature dependence of emission lifetime excited by near-ultraviolet LED. Data are provided indicating temperature and signal

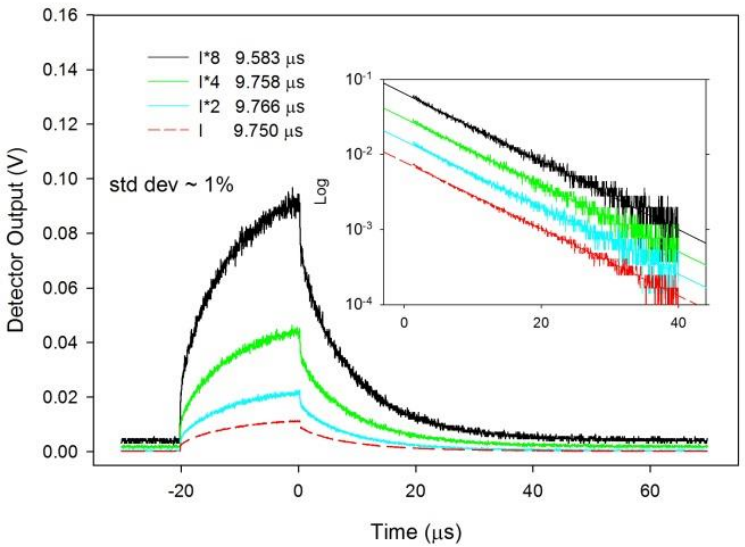

FIGURE 8. Signal for different PMT gains.

repeatability. For signal levels changing by factor of eight, the lifetime standard deviation is $+/-0.2{ }^{\circ} \mathrm{C}$. This shows detector linearity and consistent lifetime measurement capability. The Labkit may also be used to explore other areas of measurement science: the role of signal-to-noise, curve fitting, and data acquisition. The unit could also be adapted as a means to demonstrate the concept of a half-life. The advantage of this is that no radioactive material is required.

\section{REFERENCES}

1. S. W. Allison and G. T. Gillies, Review of Scientific Instruments, 68, 2615-2650 (1997).

2. C. Knappe, Lindén, J., Abou Nada, F., Richter, M., and Aldén M., Review of Scientific Instruments 83, 034901 (2012).

3. A. H. Khalid and K. Kontis, Sensors, 8, 5673-5744 (2008).

4. M. D. Chambers and D. R. Clarke, Annual Review of Materials Research, pp. 325-359 (2009).

5. M. Alden, A. Omrane, M. Richter, and G. Sarner, Progress in Energy and Combustion Science, 37, 422-461 (2011).

6. J. Brübach, C. Pflitsch, A. Dreizler, and B. Atakan, Progress in Energy and Combustion Science, 39, 37-60 (2013)

7. J. E. Parks, Temperature Dependent Lifetime Measurements of Fluorescence from a Phosphor, Special Edition for the 2013 AAPT Summer Workshop. WWW Document. (http://www. phys.utk. edu/labs/mod phys/Temperature\%20Dependent:20Lifetimes. pdf) 